\title{
MANEJO QUÍMICO DE PLANTAS ADULTAS DE DIGITARIA INSULARIS COM GLYPHOSATE ISOLADO E EM MISTURA COM CHLORIMURON-ETHYL OU QUIZALOFOP-P-TEFURIL EM ÁREA DE PLANTIO DIRETO $\left({ }^{1}\right)$
}

\author{
NÚBIA MARIA CORREIA $\left({ }^{2 *}\right)$; JULIO CEZAR DURIGAN $\left({ }^{2}\right)$
}

\begin{abstract}
RESUMO
O objetivo deste trabalho foi avaliar a eficácia do herbicida glyphosate, isolado e em mistura com chlorimuron-ethyl ou quizalofop-p-tefuril, e o efeito da adição de óleo mineral à calda de glyphosate e chlorimuron-ethy no controle de plantas adultas de Digitaria insularis, em área sob sistema de plantio direto. Foram desenvolvidos dois experimentos, em condições de campo, um de novembro de 2005 a fevereiro de 2006 e outro de novembro de 2006 a janeiro de 2007, em Jaboticabal (SP). O delineamento experimental foi o de blocos ao acaso, em esquema fatorial, com quatro repetições. No primeiro experimento, foram avaliadas oito combinações (Fator A) entre o glyphosate $(0,72 ; 1,44 ; 2,16$ e 2,88 kg $\left.\mathrm{ha}^{-1}\right)$ e o chlorimuron-ethyl (0 e $\left.10 \mathrm{~g} \mathrm{ha}^{-1}\right)$, três doses (Fator B) de óleo mineral $\left(0 ; 0,5\right.$ e 1,0 $\left.\mathrm{L} \mathrm{ha}^{-1}\right)$ e uma testemunha mantida infestada sem a aplicação de herbicida. No segundo, foram estudadas seis associações (Fator A) entre o glyphosate $\left(1,44 ; 2,16\right.$ e $\left.2,88 \mathrm{~kg} \mathrm{ha}^{-1}\right)$ e o quizalofop-p-tefuril (0 e $\left.120 \mathrm{~g} \mathrm{ha}^{-1}\right)$ e um tratamento de quizalofop-p-tefuril $\left(120 \mathrm{~g} \mathrm{ha}^{-1}\right)$ isolado, combinados com aplicações sequenciais (Fator B) de glyphosate $\left(0 ; 0,72\right.$ e $\left.1,44 \mathrm{~kg} \mathrm{ha}^{-1}\right)$, pulverizado 15 dias após a primeira a aplicação, além de uma testemunha infestada sem a aplicação de herbicida. Nas avaliações iniciais, no experimento de 2005/ 2006, em plantas pulverizadas com as maiores doses de glyphosate, isolado ou em mistura ao chlorimuron, havia injúrias visuais severas, com classificação do controle de bom $(71 \%$ a $80 \%)$ a muito bom $(81 \%$ a $90 \%)$. Com o decorrer do tempo, as plantas rebrotaram revelando seu potencial de recuperação e a ineficácia dos herbicidas no controle definitivo desta espécie. A adição de óleo mineral à calda de glyphosate e chlorimuron não contribuiu para o controle de $D$. insularis. No outro experimento, o quizalofop isolado e a sua associação ao glyphosate, combinados à aplicação sequencial de $1,44 \mathrm{~kg} \mathrm{ha}^{-1}$ de glyphosate, resultaram em melhor controle da planta daninha.
\end{abstract}

Palavras-chave: capim amargoso, cerrado, dessecação, controle químico.

\section{ABSTRACT \\ CHEMICAL MANAGEMENT OF ADULT DIGITARIA INSULARIS WITH GLYPHOSATE ALONE AND MIXTURE WITH CHLORIMURON-ETHYL AND QUIZALOFOP-P-TEFURIL IN A NO-TILLAGE FIELD}

\begin{abstract}
The objective of this work was to evaluate the efficiency of the herbicide glyphosate used alone, in combination with chlorimuron-ethyl and quizalofop-p-tefuril as well as the effect of the addition of mineral oil to control adult Digitaria insularis plants in a no-tillage field. Two experiments were conducted under field conditions from November 2005 to February 2006 and from November 2006 to January 2007, at Jaboticabal, São Paulo State, Brazil. Experiments were set in randomized blocks with four replications. In the first experiment $(2005 / 06)$ it were tested eight combinations among glyphosate $(0.72 ; 1.44 ; 2.16$ and $\left.2.88 \mathrm{~kg} \mathrm{ha}^{-1}\right)$, chlorimuron-ethyl $\left(0\right.$ and $\left.10 \mathrm{~g} \mathrm{ha}^{-1}\right)$ mineral oil $\left(0 ; 0.5\right.$ and $\left.1.0 \mathrm{~L} \mathrm{ha}^{-1}\right)$ and an infested, untreated control. In the second experiment (2006/07) it were tested six associations among glyphosate
\end{abstract}

$\left.{ }^{1}\right)$ Recebido para publicação em $1 .^{\circ}$ de abril de 2008 e aceito em 24 de abril de 2009.

$\left(^{2}\right)$ Departamento de Fitossanidade, Universidade Estadual Paulista (UNESP). Campus de Jaboticabal, Via de Acesso Prof. Paulo Donato Castellane, s/n., 14884-900 Jaboticabal (SP). Brasil. E-mail: correianm@fcav.unesp.br. *Autora correspondente. 
(1.44; 2.16 and $\left.2.88 \mathrm{~kg} \mathrm{ha}^{-1}\right)$, quizalofop-p-tefuril (0 and $\left.120 \mathrm{~g} \mathrm{ha}^{-1}\right)$, quizalofop-p-tefuril $\left(120 \mathrm{~g} \mathrm{ha}^{-1}\right)$ alone and combined to three $\left(0 ; 0.72\right.$ and $\left.1.44 \mathrm{~kg} \mathrm{ha}^{-1}\right)$ glyphosate doses, sprayed 15 days after the first application, besides an infested and untreated control. In 2005/06, plants sprayed with higher glyphosate doses, alone or combined with chlorimuron, showed severe injuries, with a control classification ranging from good (71 to $80 \%$ ) to very good ( 81 to $90 \%$ ). Over time plants tillered and showed their recovery potential and the inefficiency of the herbicides to control this weed species. The addition of mineral oil to glyphosate and chlorimuron solution did not entrance its efficiency. In the other experiment, the quizalofop alone or associated with glyphosate, combined to sequential application of glyphosate $1.44 \mathrm{~kg} \mathrm{ha}^{-1}$, resulted in the best weed control.

Key words: sourgrass, Brazilian cerrado, desiccation, chemical control.

\section{INTRODUÇÃO}

O sistema de produção denominado plantio direto tem como operação principal a dessecação ou manejo químico das plantas, que consiste na aplicação ou na mistura de dois ou mais herbicidas, ou ainda de aplicações sequenciais, antes da semeadura das culturas (Procópio et al., 2006b). A escolha de um tipo de manejo ocorre em função das espécies de plantas daninhas presentes na área, do nível de infestação, das condições climáticas e edáficas e da cultura a ser cultivada (ProcópIo et al., 2006a).

Nas áreas onde não há o estabelecimento de culturas de cobertura na entressafra tem-se observado aumento na infestação de capim-amargoso (Digitaria insularis (L.) Fedde). A espécie é uma planta perene, herbácea, ereta, de colmos estriados e com 50 a 100 cm de altura (KISSMANN e GROTH, 1997). Forma touceiras a partir de curtos rizomas, mas se reproduz também por semente. Possui grande potencial como infestante, pois suas sementes, revestidas por muitos pelos, são carregadas pelo vento a grandes distâncias, com bom poder germinativo (KISSMANN e GROTH, 1997).

O crescimento inicial das plantas de $D$. insularis, principalmente em condições de sombreamento ou de baixas temperaturas, pode ser suprimido pelo crescimento mais rápido de outras espécies da comunidade infestante (MACHADO et al., 2006). Esse processo é causado pelo seu crescimento inicial lento até os 45 dias. No entanto, se a dose do herbicida não for suficiente para controlá-la, pode se tornar uma espécie dominante, como ocorre em muitos casos em áreas de café, citros e plantio direto, onde a aplicação de glyphosate é eficaz para outras espécies e insatisfatória no controle de D. insularis (MACHADO et al., 2006).

Ao determinar a curva de dose-resposta de $D$. insularis ao herbicida glyphosate, LACERDA e VICTÓRIA FILHO (2004) verificaram que houve sensibilidade intermediária dessa espécie às doses do produto e redução expressiva do crescimento na dose de $1,44 \mathrm{~kg}$ $\mathrm{ha}^{-1}$. Os resultados foram atingidos com aplicações de glyphosate em plântulas com apenas quatro folhas, antes do seu perfilhamento. Em observações de campo, em áreas onde há uso contínuo de glyphosate, constatou-se que plantas originárias de sementes, quando jovens, são controladas pelo herbicida (MACHADO et al., 2006); contudo, quando se desenvolvem e formam rizomas, seu controle é ineficaz. Para um controle mais eficaz, a aplicação de glyphosate deveria ser realizada até os 35 dias após a emergência, quando os rizomas ainda não foram formados (MACHADO et al., 2006).

Uma estratégia de manejo químico de plantas adultas de $D$. insularis, em áreas de plantio direto, é a combinação de outros herbicidas ao glyphosate. Procópio et al. (2006a), ao avaliar a eficácia de glyphosate e da mistura comercial paraquat + diuron, e o efeito do intervalo entre as aplicações dos herbicidas e a semeadura da soja, no controle e rebrota de $D$. insularis, relataram que para maior eficácia no controle, a aplicação de glyphosate $\left(1,80 \mathrm{~kg} \mathrm{ha}^{-1}\right)$ deveria ser feita pelo menos dois dias antes da semeadura da soja. Esse resultado não diferiu do controle obtido com as aplicações sequenciais de glyphosate e paraquat + diuron. Houve menor porcentagem de rebrota nos tratamentos que receberam a aplicação de glyphosate seguida de paraquat + diuron, independentemente do intervalo de 10, 15 e 20 dias entre eles, e no tratamento em que se efetuou apenas uma aplicação de glyphosate, cinco dias antes da semeadura.

O objetivo deste trabalho foi avaliar a eficácia do herbicida glyphosate, isolado e em mistura com chlorimuron-ethyl ou quizalofop-p-tefuril, e o efeito da adição de óleo mineral à calda de glyphosate e chlorimuron-ethyl no controle de plantas adultas de D. insularis, em área de plantio direto.

\section{MATERIAL E MÉTODOS}

Foram desenvolvidos dois experimentos, em condições de campo - o primeiro de novembro de 2005 a fevereiro de 2006 e o outro de novembro de 2006 a janeiro de 2007, em Jaboticabal (SP). A área experimental apresentava alta infestação de $D$. 
insularis (em torno de 95\%) e estava uniformemente coberta pela planta daninha no momento da aplicação dos herbicidas.

O delineamento experimental foi o de blocos ao acaso em ambos os experimentos, em esquema fatorial $8 \times 3+1$ (no primeiro) e $7 \times 3+1$ (no segundo), com quatro repetições. No experimento de 2005/2006, foram avaliadas oito combinações (Fator A) entre o glyphosate $\left(0,72 ; 1,44 ; 2,16\right.$ e $\left.2,88 \mathrm{~kg} \mathrm{ha}^{-1}\right)$ e o chlorimuron-ethyl ( 0 e $\left.10 \mathrm{~g} \mathrm{ha}^{-1}\right)$, três doses (Fator B) de óleo mineral $\left(0 ; 0,5\right.$ e 1,0 $\left.\mathrm{L} \mathrm{ha}^{-1}\right)$ e uma testemunha mantida infestada sem a aplicação de herbicida. Em 2006/2007, foram estudadas seis associações (Fator A) entre o glyphosate $\left(1,44 ; 2,16\right.$ e $\left.2,88 \mathrm{~kg} \mathrm{ha}^{-1}\right)$ e o quizalofop-p-tefuril ( 0 e $\left.120 \mathrm{~g} \mathrm{ha}^{-1}\right)$ e um tratamento de quizalofop-p-tefuril (120 $\mathrm{g} \mathrm{ha}^{-1}$ ) sozinho, combinados com a aplicação sequencial (Fator B) de glyphosate $\left(0 ; 0,72\right.$ e 1,44 $\left.\mathrm{kg} \mathrm{ha}^{-1}\right)$, pulverizado 15 dias após a primeira a aplicação. Também foi mantida uma testemunha infestada sem a aplicação de herbicida.

As parcelas mediam 2,0 m de largura e 4,0 m de comprimento, totalizando $8,0 \mathrm{~m}^{2}$. Como área útil consideraram-se os $3,0 \mathrm{~m}^{2}$ centrais $(1,0 \mathrm{~m} \mathrm{x} 3,0 \mathrm{~m})$.

No momento da pulverização, as plantas de capim-amargoso estavam em pleno desenvolvimento reprodutivo (produção de sementes), com altura média de $1,0 \mathrm{~m}$, considerando-se a distância do colo da planta até a extremidade da panícula, ou de $0,7 \mathrm{~m}$ do colo à inserção da última folha, no primeiro experimento. No segundo, esses valores foram de 1,6 e $1,0 \mathrm{~m}$ respectivamente.

Os herbicidas e o óleo mineral, nas diferentes doses e misturas, foram aplicados utilizando-se de pulverizador costal, à pressão constante (mantida por $\mathrm{CO}_{2}$ comprimido) de $1,9 \mathrm{kgf} \mathrm{cm}{ }^{-2}$, munido de barra com quatro bicos de jato plano leque XR11002, espaçados de $0,5 \mathrm{~m}$, com consumo de calda equivalente a $150 \mathrm{~L} \mathrm{ha}^{-1}$. No momento da aplicação, constatou-se, em 2005/2006 e 2006/2007, respectivamente, $29,3{ }^{\circ} \mathrm{C}$ e $26,4^{\circ} \mathrm{C}$ de temperatura do ar; $25,4{ }^{\circ} \mathrm{C}$ e $26,4{ }^{\circ} \mathrm{C}$ de temperatura do solo (a $5 \mathrm{~cm}$ de profundidade); $63 \%$ e $56 \%$ de umidade relativa do ar; de 2 a $5 \mathrm{~km} \mathrm{~h}^{-1}$ e de 3 a $6 \mathrm{~km} \mathrm{~h}^{-1}$ de velocidade do vento e solo na capacidade de campo.

No primeiro experimento, foram realizadas avaliações visuais de controle aos 14, 21, 28 e 42 dias após a aplicação (DAA) dos herbicidas, utilizandose escala de notas de 0 a 100\%, em que zero representa a ausência de injúrias visuais e 100 a morte da planta. Aos 42 DAA, a área experimental foi roçada para eliminação da parte aérea das plantas e estimulação de rebrota. Após 42 dias do corte, utilizando-se quadro com $0,45 \mathrm{~m}^{2}$ de área interna, depositado aleatoriamente na área útil das parcelas, fez-se a coleta da parte aérea da rebrota das plantas. $O$ material foi seco em estufa a $50{ }^{\circ} \mathrm{C}$, até atingir massa constante, quando foi avaliado.

No segundo experimento, as avaliações de controle (em porcentagem) foram realizadas aos $21,28 \mathrm{e}$ 35 dias após a primeira aplicação (DAPA) dos herbicidas. Aos 42 DAA, utilizando-se quadro com $0,45 \mathrm{~m}^{2}$ de área interna, depositado aleatoriamente na área útil das parcelas, fez-se a coleta de toda a parte aérea verde das plantas. $\mathrm{O}$ material foi seco em estufa a $50^{\circ} \mathrm{C}$, até atingir massa constante, quando foi avaliado.

Os resultados foram submetidos ao teste $\mathrm{F}$ da análise de variância. Os efeitos dos herbicidas (glyphosate, chlorimuron e quizalofop), do óleo mineral e da aplicação sequencial, quando significativos, foram comparados pelo teste de Tukey a $5 \%$ de probabilidade. O programa estatístico utilizado foi o SISVAR (FERREIRA, 1999).

Como as notas das avaliações visuais de controle foram estabelecidas em função das parcelas testemunhas, não houve a necessidade de inclusão deste tratamento na análise estatística. Assim, optouse pela sua exclusão, obteve-se, com isto, melhor distribuição e homogeneidade das notas de controle, não justificando uma possível transformação dos dados. Para matéria seca da parte aérea, o desenvolvimento da testemunha sem aplicação foi avaliado em relação aos tratamentos de herbicidas, segundo um esquema de contrastes ortogonais de interesse. Aplicou-se o teste F para cada contraste, aceitando-se ou rejeitando-se a hipótese H0 (Y=0).

\section{RESULTADOS E DISCUSSÃO}

Eficácia de glyphosate, isolado e em mistura com chlorimuron-ethyl, com e sem a adição de óleo mineral à calda

Nas avaliações visuais realizadas aos 14,21 e 28 DAA (Tabelas 1 e 2), o melhor controle de $D$. insularis foi obtido com a aplicação das maiores doses de glyphosate $\left(2,16\right.$ e $\left.2,88 \mathrm{~kg} \mathrm{ha}^{-1}\right)$, isoladas ou associadas ao chlorimuron. Independentemente da adição de óleo mineral à calda, o chlorimuron não contribuiu significativamente para o controle da planta daninha. De modo geral, o uso de óleo mineral associado aos herbicidas não melhorou sua eficácia. PROCópio et al. (2006b) constataram que a adição dos herbicidas imazethapyr (100 $\left.\mathrm{g} \mathrm{ha}^{-1}\right)$ ou de chlorimuron-ethyl (10 ou $20 \mathrm{~g} \mathrm{ha}^{-1}$ ) à calda de glyphosate $\left(1,62 \mathrm{~kg} \mathrm{ha}^{-1}\right)$ não aumentou o controle de D. insularis, com resultados similares à aplicação de glyphosate sozinho. O mesmo comportamento foi observado para a porcentagem de rebrota. 


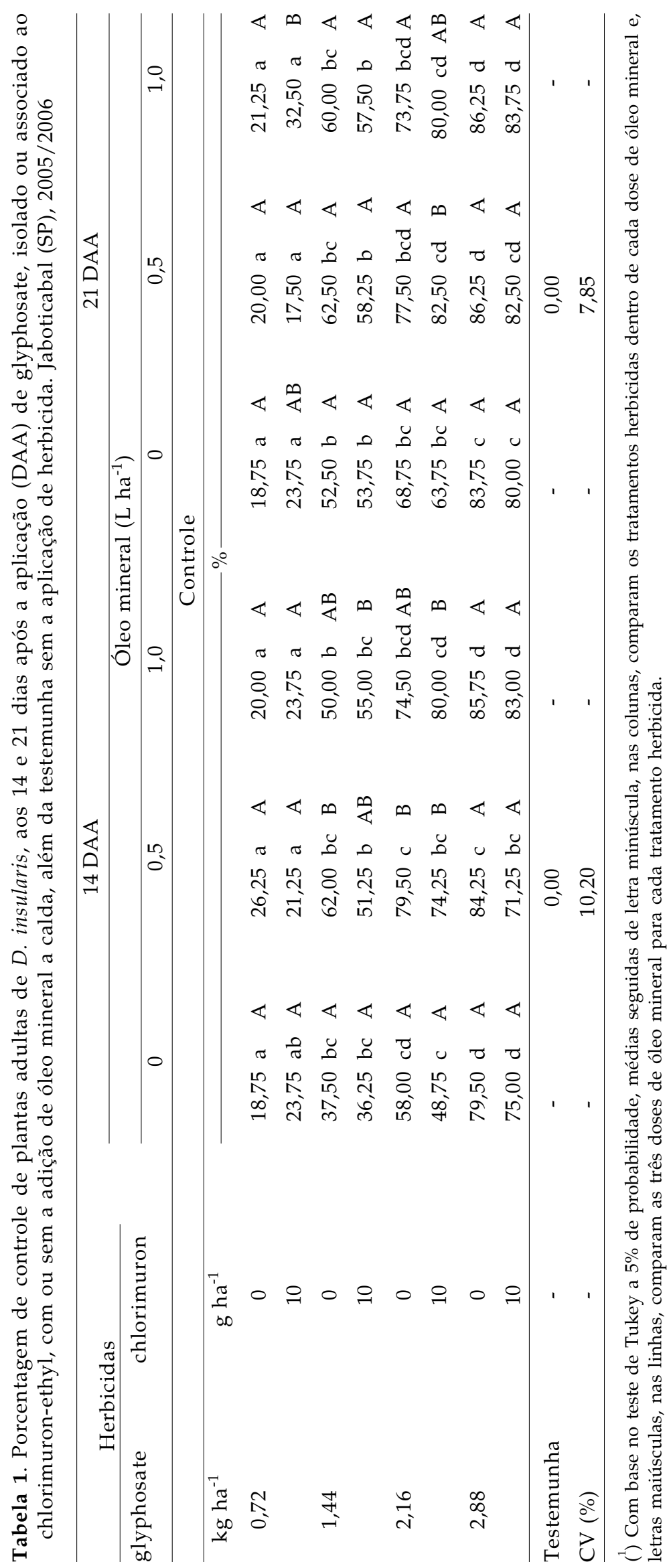




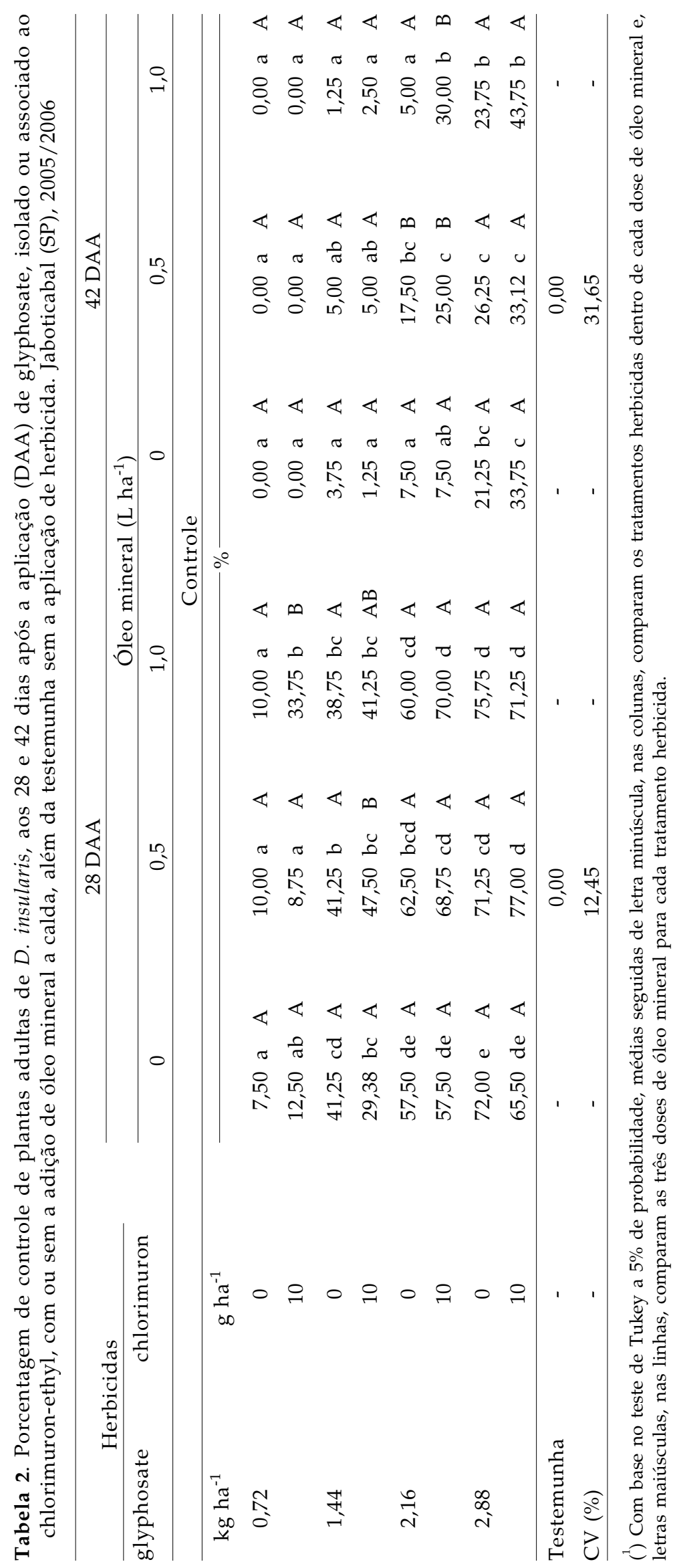


Aos 42 DAA (Tabela 2) observou-se início da rebrota das plantas de D. insularis, comprovando sua capacidade de recuperação e ineficácia dos herbicidas e de suas misturas no controle. Motivo, pelos quais, as notas de controle foram menores, comparadas às avaliações anteriores. A porcentagem média atribuída à associação de $2,88 \mathrm{~kg} \mathrm{ha}^{-1} \mathrm{de}$ glyphosate com $10 \mathrm{~g}$ de chlorimuron foi $37 \%$, considerada como nenhum controle, segundo a escala da Alam (1974).

Aos 42 dias após o corte das plantas, verificou-se que não houve efeito significativo da adição de óleo mineral à calda na matéria seca da rebrota (Tabela 3). Sem o aditivo, o menor acúmulo de massa foi constatado com as aplicações de 0,72 ; 2,16 e $2,88 \mathrm{~kg} \mathrm{ha}^{-1}$ de glyphosate, isolado e em mistura, e da associação de $1,44 \mathrm{~kg} \mathrm{ha}^{-1}$ deste herbicida ao chlorimuron. Quando utilizado 0,5 L ha $^{-1}$ de óleo mineral, os melhores resultados foram obtidos com a aplicação das três maiores doses de glyphosate, isoladas ou associadas ao chlorimuron. O mesmo ocorreu com a adição 1,0 L $\mathrm{ha}^{-1}$ de óleo mineral à calda, incluindo o tratamento de $0,72 \mathrm{~kg} \mathrm{ha}^{-1}$ de glyphosate em mistura com $10 \mathrm{~g}$ de chlorimuron.

Tabela 3. Matéria seca da rebrota de plantas de D. insularis, pulverizadas com glyphosate, isolado ou associado ao chlorimuron-ethyl, com ou sem a adição de óleo mineral a calda, aos 42 dias após o corte da parte aérea das plantas, além da testemunha sem a aplicação de herbicida, Jaboticabal (SP), 2005/2006

\begin{tabular}{|c|c|c|c|c|}
\hline \multicolumn{2}{|c|}{ Herbicidas } & \multicolumn{3}{|c|}{ Óleo mineral $\left(\mathrm{L} \mathrm{ha}^{-1}\right)$} \\
\hline \multirow[t]{2}{*}{ Glyphosate } & \multirow[t]{2}{*}{ Chlorimuron } & 0 & 0,5 & 1,0 \\
\hline & & \multicolumn{3}{|c|}{ Matéria seca } \\
\hline $\mathrm{kg} \mathrm{ha}{ }^{-1}$ & $\mathrm{~g} \mathrm{ha}^{-1}$ & 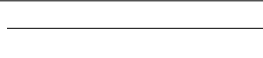 & $\mathrm{g} \mathrm{m}^{-2}$ & 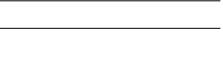 \\
\hline \multirow[t]{2}{*}{0,72} & 0 & 114,24 ab $\mathrm{A}\left({ }^{1}\right)$ & 134,68 bc A & $142,22 \mathrm{~b} \quad \mathrm{~A}$ \\
\hline & 10 & $127,09 \mathrm{ab} A$ & 181,53 с & 79,88 ab $\mathrm{AB}$ \\
\hline \multirow[t]{2}{*}{1,44} & 0 & $145,76 \mathrm{~b} \quad \mathrm{~A}$ & 105,70 abc A & $90,37 \mathrm{ab} A$ \\
\hline & 10 & 77,52 ab $A$ & 100,37 abc A & 52,72 ab A \\
\hline \multirow[t]{2}{*}{2,16} & 0 & 37,60 a $A$ & $50,27 \mathrm{ab} \quad \mathrm{A}$ & 89,13 ab A \\
\hline & 10 & $96,94 \mathrm{ab} A$ & 58,88 ab A & $52,32 \mathrm{ab} A$ \\
\hline \multirow[t]{2}{*}{2,88} & 0 & 41,57 a $\mathrm{A}$ & 35,74 a $\mathrm{A}$ & 88,93 ab A \\
\hline & 10 & 42,23 a $\mathrm{A}$ & 45,53 ab A & 25,70 a $A$ \\
\hline Testemunha & - & - & 199,90 & - \\
\hline CV (\%) & - & - & 51,81 & - \\
\hline
\end{tabular}

$\left({ }^{1}\right)$ Com base no teste de Tukey a 5\% de probabilidade, médias seguidas de letra minúscula, nas colunas, comparam os tratamentos herbicidas dentro de cada dose de óleo mineral e, letras maiúsculas, nas linhas, comparam as três doses de óleo mineral para cada tratamento herbicida.

Por meio da análise de contraste, observou-se que a testemunha sem aplicação diferiu significativamente dos tratamentos 2,16 e 2,88 de kg $\mathrm{ha}^{-1}$ de glyphosate isolado e em mistura com $0,5 \mathrm{~L} \mathrm{ha}^{-}$ 1 de óleo mineral; 2,88 de $\mathrm{kg} \mathrm{ha}^{-1}$ de glyphosate associado ao chlorimuron sem ou com a adição de 0,5 $\mathrm{L} \mathrm{ha}^{-1}$ de óleo mineral; 1,$44 ; 2,16$ e 2,88 de $\mathrm{kg} \mathrm{ha}^{-1} \mathrm{de}$ glyphosate em mistura com chlorimuron e 1,0 $\mathrm{L} \mathrm{ha}^{-1}$ de óleo mineral, os quais resultaram em menor acúmulo de massa nas plantas.

Apesar dos resultados em avaliações iniciais de controle e na matéria seca da rebrota, a associação entre os herbicidas e o óleo mineral não ocasionou controle satisfatório de $D$. insularis, exigindo outras estratégias de manejo, como o emprego de manejo mecânico da planta associado ao uso de herbicidas; a aplicação sequencial de glyphosate; a associação ou a combinação de glyphosate a outro herbicida e/ou a pulverização antes da formação de rizomas na planta.

Eficácia de glyphosate, isolado e em mistura ao quizalofop-p-tefuril, combinados à aplicação sequencial de glyphosate

Aos 21 e 28 DAA (Tabelas 4 e 5), as associações de quizalofop às três doses de glyphosate resultaram em melhor controle de $D$. insularis, quando comparado às aplicações isoladas de glyphosate. Com a aplicação de quizalofop sozinho o controle foi estatisticamente similar às suas misturas com glyphosate. Esses resultados foram constatados nas três combinações com a aplicação sequencial de glyphosate. 
Tabela 4. Porcentagem de controle de plantas adultas de D. insularis, aos 21 dias após a aplicação (DAA) de glyphosate e quizalofop-p-tefuril (isolados e em associação), combinados com a aplicação sequencial de glyphosate, realizada 15 dias após a primeira, além da testemunha sem a aplicação de herbicida. Jaboticabal (SP), 2006/2007

\begin{tabular}{|c|c|c|c|c|}
\hline \multicolumn{2}{|c|}{ Herbicidas } & \multicolumn{3}{|c|}{ Aplicação sequencial de glyphosate $\left(\mathrm{kg} \mathrm{ha}^{-1}\right)$} \\
\hline \multirow[t]{2}{*}{ glyphosate } & quizalofop & 0 & 0,72 & 1,44 \\
\hline & & \multicolumn{3}{|c|}{ Controle } \\
\hline $\mathrm{kg} \mathrm{ha}^{-1}$ & $\mathrm{~g} \mathrm{ha}^{-1}$ & 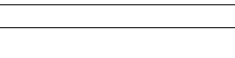 & $\%$ & +2 \\
\hline 0 & 120 & 73,50 a $\mathrm{A}\left(\left(^{1}\right)\right.$ & $73,75 \mathrm{ab} A$ & 85,00 a $\mathrm{A}$ \\
\hline \multirow[t]{2}{*}{1,44} & 0 & 0,00 с $C$ & $22,50 \mathrm{~d} \quad \mathrm{~B}$ & 61,25 b A \\
\hline & 120 & 72,50 a $\mathrm{A}$ & 62,50 bc $\mathrm{A}$ & $76,25 \mathrm{ab} A$ \\
\hline \multirow[t]{2}{*}{2,16} & 0 & 22,50 c B & 48,75 с $\mathrm{A}$ & $60,00 \quad b \quad A$ \\
\hline & 120 & 82,50 a $\mathrm{A}$ & 81,25 ab A & 90,00 a $\mathrm{A}$ \\
\hline \multirow[t]{2}{*}{2,88} & 0 & 47,50 b $\quad$ B & 63,75 bc $\mathrm{AB}$ & 72,50 ab A \\
\hline & 120 & 81,25 a $A$ & 87,50 a $\mathrm{A}$ & 91,25 a $\mathrm{A}$ \\
\hline Testemunha & - & - & 0,00 & - \\
\hline CV (\%) & - & - & 16,31 & - \\
\hline
\end{tabular}

$\left({ }^{1}\right)$ Com base no teste de Tukey a 5\% de probabilidade, médias seguidas de letra minúscula, nas colunas, comparam os tratamentos herbicidas dentro de cada dose de glyphosate (em aplicação sequencial) e, letras maiúsculas, nas linhas, comparam as três doses de glyphosate (em aplicação sequencial) para cada tratamento herbicida.

Tabela 5. Porcentagem de controle de plantas adultas de D. insularis, aos 28 dias após a aplicação (DAA) de glyphosate e quizalofop-p-tefuril (isolados e em associação), combinados com a aplicação sequencial de glyphosate, realizada 15 dias após a primeira, além da testemunha sem a aplicação de herbicida. Jaboticabal (SP), 2006/2007

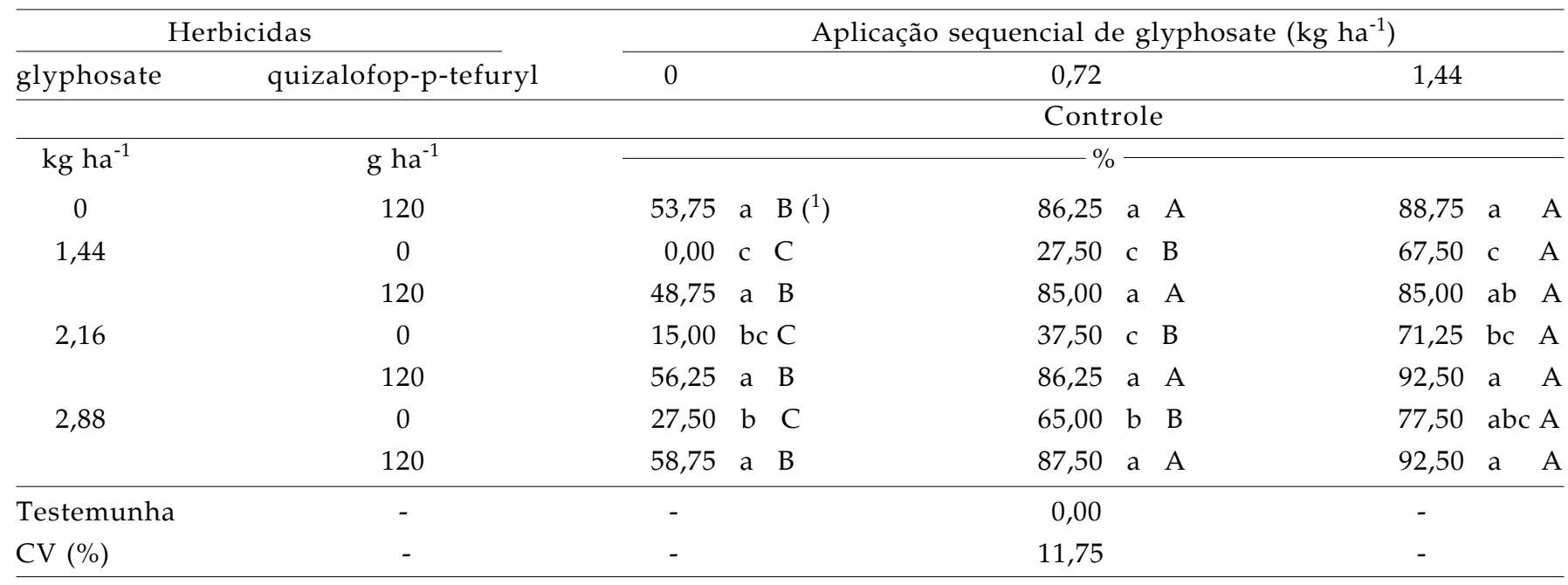

$\left.{ }^{1}\right)$ Com base no teste de Tukey a 5\% de probabilidade, médias seguidas de letra minúscula, nas colunas, comparam os tratamentos herbicidas dentro de cada dose de glyphosate (em aplicação sequencial) e, letras maiúsculas, nas linhas, comparam as três doses de glyphosate (em aplicação sequencial) para cada tratamento herbicida.

Aos 21 DAA, a aplicação sequencial, independentemente da dose de glyphosate utilizada, não controlou $D$. insularis quando o glyphosate foi associado ao quizalofop na primeira aplicação (Tabela 4). O mesmo foi observado com a aplicação de quizalofop apenas. Contudo, aos 28 DAA, a aplicação sequencial contribuiu para maior eficácia de controle de $D$. insularis. Para as associações de quizalofop mais glyphosate não houve diferença significativa entre as doses de 0,72 e $1,44 \mathrm{~kg} \mathrm{ha}^{-1}$ de glyphosate aplicado em sequência. Quando aplicado o glyphosate sozinho, a dose de $1,44 \mathrm{~kg}$ $\mathrm{ha}^{-1}$ foi mais eficaz que a de $0,72 \mathrm{~kg} \mathrm{ha}^{-1}$. SchmidT et al. (2006) relataram que o glyphosate $(0,72$ e 1,44 $\left.\mathrm{kg} \mathrm{ha}^{-1}\right)$, o clethodim (96 $\mathrm{g} \mathrm{ha}^{-1}$ ) e o tepraloxydim (80 e $100 \mathrm{~g} \mathrm{ha}^{-1}$ ) foram eficazes no controle de $D$. insularis, quando pulverizados em plantas com um a três perfilhos. 
Aos 35 DAA (Tabela 6), constatou-se início de rebrota na maioria das plantas de $D$. insularis com morte parcial ou total da parte aérea. O efeito foi mais pronunciado nos tratamentos sem aplicação sequencial de glyphosate, cujo controle foi muito baixo. O quizalofop isolado e sua associação ao glyphosate $\left(1,44 ; 2,16\right.$ e $\left.2,88 \mathrm{~kg} \mathrm{ha}^{-1}\right)$, combinados à aplicação sequencial de $1,44 \mathrm{~kg} \mathrm{ha}^{-1}$ de glyphosate, resultaram em melhor controle de $D$. insularis.
Para matéria seca da parte aérea, a interação dos fatores (herbicida e aplicação sequencial) não foi significativa (Tabela 7), indicando que se comportaram de forma independente para esta variável. Nas plantas tratadas com as misturas de quizalofop mais glyphosate e quizalofop sozinho houve redução média de $75 \%$ no acúmulo de matéria seca, em relação à testemunha sem herbicida.

Tabela 6. Porcentagem de controle de plantas adultas de D. insularis, aos 35 dias após a aplicação (DAA) de glyphosate e quizalofop-p-tefuril (isolados e em associação), combinados com a aplicação sequencial de glyphosate, realizada 15 dias após a primeira, além da testemunha sem a aplicação de herbicida, Jaboticabal (SP), $2006 / 2007$

\begin{tabular}{|c|c|c|c|c|c|c|}
\hline \multicolumn{2}{|c|}{ Herbicidas } & \multicolumn{5}{|c|}{ Aplicação sequencial de glyphosate $\left(\mathrm{kg} \mathrm{ha}^{-1}\right)$} \\
\hline \multirow[t]{2}{*}{ glyphosate } & \multirow[t]{2}{*}{ quizalofop-p-tefuryl } & \multicolumn{2}{|r|}{0} & 0,72 & \multicolumn{2}{|c|}{1,44} \\
\hline & & \multicolumn{5}{|c|}{ Controle } \\
\hline $\mathrm{kg} \mathrm{ha}^{-1}$ & $\mathrm{~g} \mathrm{ha}^{-1}$ & & 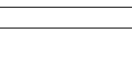 & $\%$ & & \\
\hline 0 & 120 & 10,00 & a $C\left({ }^{1}\right)$ & 47,50 a $B$ & 77,50 a & a $\mathrm{A}$ \\
\hline \multirow[t]{2}{*}{1,44} & 0 & 0,00 & a $\mathrm{C}$ & $20,00 \mathrm{~cd} \mathrm{~B}$ & 45,00 & b A \\
\hline & 120 & 10,00 & a $C$ & $43,75 \mathrm{ab} \mathrm{B}$ & 65,00 a & a $\mathrm{A}$ \\
\hline \multirow[t]{2}{*}{2,16} & 0 & 2,50 & a $\mathrm{B}$ & 17,50 d A & 28,75 c & c A \\
\hline & 120 & 10,00 & a $C$ & 46,25 ab $B$ & 76,25 a & a A \\
\hline \multirow[t]{2}{*}{2,88} & 0 & 0,00 & a C & 32,50 bc $B$ & $47,50 \mathrm{~b}$ & b A \\
\hline & 120 & 12,50 & a C & 55,00 a $\mathrm{B}$ & 73,75 a & a $\mathrm{A}$ \\
\hline Testemunha & - & - & & 0,00 & - & \\
\hline CV (\%) & - & - & & 19,84 & - & \\
\hline
\end{tabular}

$\left({ }^{1}\right)$ Com base no teste de Tukey a 5\% de probabilidade, médias seguidas de letra minúscula, nas colunas, comparam os tratamentos herbicidas dentro de cada dose de glyphosate (em aplicação sequencial) e, letras maiúsculas, nas linhas, comparam as três doses de glyphosate (em aplicação sequencial) para cada tratamento herbicida.

Tabela 7. Matéria seca de plantas ou da rebrota de D. insularis, aos 49 dias após a aplicação (DAA) de glyphosate e quizalofopp-tefuril (isolados e em associação), combinados com a aplicação sequencial de glyphosate, realizada 15 dias após a primeira, além da testemunha sem a aplicação de herbicida. Jaboticabal (SP), 2006/2007.

\begin{tabular}{|c|c|c|c|c|}
\hline \multicolumn{2}{|c|}{ Herbicidas } & \multicolumn{3}{|c|}{ Matéria seca } \\
\hline glyphosate & quizalofop & & & Redução \\
\hline $\mathrm{kg} \mathrm{ha}^{-1}$ & $\mathrm{~g} \mathrm{ha}^{-1}$ & $\mathrm{~g} \mathrm{~m}^{-2}$ & & $\%$ \\
\hline 0 & 120 & 268,15 & a $\left({ }^{1}\right)$ & 75,87 \\
\hline \multirow[t]{2}{*}{1,44} & 0 & 587,78 & $\mathrm{~b}$ & 47,10 \\
\hline & 120 & 302,59 & a & 72,77 \\
\hline \multirow[t]{2}{*}{2,16} & 0 & 516,67 & $\mathrm{~b}$ & 46,50 \\
\hline & 120 & 259,07 & a & 76,68 \\
\hline \multirow[t]{2}{*}{2,88} & 0 & 477,04 & $\mathrm{~b}$ & 57,07 \\
\hline & 120 & 273,70 & a & 75,37 \\
\hline \multicolumn{5}{|c|}{ Aplicação sequencial de glyphosate } \\
\hline \multicolumn{5}{|c|}{$\mathrm{kg} \mathrm{ha}^{-1}$} \\
\hline \multicolumn{2}{|c|}{0} & 513,49 & $\mathrm{~b}$ & 53,79 \\
\hline \multicolumn{2}{|c|}{0,72} & 360,24 & a & 67,58 \\
\hline \multicolumn{2}{|c|}{1,44} & 276,98 & a & 75,07 \\
\hline Testemunha & & 1111,11 & & 0,00 \\
\hline CV (\%) & & 34,13 & & - \\
\hline
\end{tabular}

$\left({ }^{1}\right)$ Médias seguidas da mesma letra não diferem significativamente entre si pelo teste de Tukey a 5\% de probabilidade. 
Para aquelas pulverizadas com as três doses de glyphosate isolado, o valor médio foi de $50 \%$. Quanto às aplicações sequenciais, não houve diferença significativa entre as doses de 0,72 e 1,44 $\mathrm{kg} \mathrm{ha}^{-1}$ de glyphosate; no entanto, elas diferiram do tratamento sem a segunda aplicação.

Por meio da comparação por contrastes, constatou-se que a testemunha não tratada não diferiu apenas da aplicação das três doses de glyphosate isolado sem a realização de aplicação sequencial.

\section{CONCLUSÕES}

1. A associação de glyphosate ao chlorimuronethyl e a adição de óleo mineral à calda dos herbicidas é ineficaz no controle de $D$. insularis.

2. O quizalofop isolado e a sua associação ao glyphosate, combinados à aplicação sequencial de $1,44 \mathrm{~kg} \mathrm{ha}^{-1}$ de glyphosate, resultam em melhor controle de $D$. insularis.

\section{REFERÊNCIAS}

ALAM - Associacion Latinoamericana de Malezas. Recomendaciones sobre unificacion de los sistemas de evaluacion em ensayos de control de malezas. ALAM, v.1, p.35-38, 1974.

FERREIRA, D.F. Sistema de análise de variância (SIRVAR). Versão 4.6. Lavras: Universidade Federal de Lavras, 1999. CDROM.

KISSMANN, K.G.; GROTH, D. Plantas infestantes e nocivas. São Paulo: BASF, 2.ed., 1997. 825p. (Tomo I)

LACERDA, A.L.S.; VICTÓRIA FILHO, R. Curvas dose-resposta em espécies de plantas daninhas com o uso do herbicida glyphosate. Bragantia, v.63, p.73-79, 2004.

MACHADO, A. F. L.; FERREIRA, L. R.; FERREIRA, F. A.; FIALHO, C. M. T.; TUFFI SANTOS, L. D.; MACHADO, M. S. Análise do crescimento de Digitaria insularis. Planta Daninha, v.24, p.641-647, 2006.

PROCÓPIO, S. O.; PIRES, F.R.; MENEZES, C. C. E.; BARROSO, A. L. L.; MORAES, R. V.; SILVA, M. V. V.; QUEIROZ, R. G.; CARMO, M. L. Efeitos de dessecantes no controle de plantas daninhas na cultura da soja. Planta Daninha, v.24, p.193-197, 2006a.

PROCÓPIO, S. O.; MENEZES, C. C. E.; PIRES, F.R.; BARROSO, A. L. L.; CARGNELUTTI FILHO, A.; RUDOVALHO, M. C.; MORAES, R. V.; SILVA, M. V. V.; CAETANO, J. O. Eficácia de imazethapyr e chloriuron-ethyl em aplicações de présemeadura da cultura da soja. Planta Daninha, v.24, p.467-473, $2006 b$.
SCHMIDT, D. F.; ROCHA, M. G.; NICOLAI, M.; MOREIRA, M. S.; CHRISTOFFOLETI, P. J. Controle de capim-amagoso (Digitaria insularis) com o uso de herbicidas pós-emergentes da cultura da soja. SIMPÓSIO INTERNACIONAL DE INICIAÇÃO CIENTÍFICA DA UNIVERSIDADE DE SÃO PAULO, 14., Piracicaba, SP. Resumos... Piracicaba: USP, 2006. [CD-ROM] 